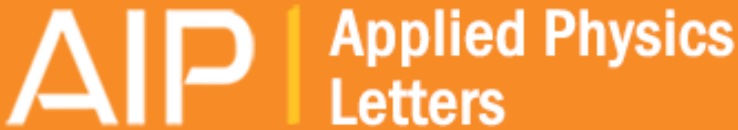

Tracking the density evolution in counter-propagating shock waves using imaging $X$ ray scattering

U. Zastrau, E. J. Gamboa, D. Kraus, J. F. Benage, R. P. Drake, P. Efthimion, K. Falk, R. W. Falcone, L. B. Fletcher, E. Galtier, M. Gauthier, E. Granados, J. B. Hastings, P. Heimann, K. Hill, P. A. Keiter, J. Lu, M. J. MacDonald, D. S. Montgomery, B. Nagler, N. Pablant, A. Schropp, B. Tobias, D. O. Gericke, S. H. Glenzer, and H. J. Lee

Citation: Applied Physics Letters 109, 031108 (2016); doi: 10.1063/1.4959256

View online: http://dx.doi.org/10.1063/1.4959256

View Table of Contents: http://scitation.aip.org/content/aip/journal/apl/109/3?ver=pdfcov

Published by the AIP Publishing

\section{Articles you may be interested in}

Combined x-ray scattering, radiography, and velocity interferometry/streaked optical pyrometry measurements of warm dense carbon using a novel technique of shock-and-releasea)

Phys. Plasmas 21, 056309 (2014); 10.1063/1.4876613

Development of a graphite polarization analyzer for resonant inelastic x-ray scattering

Rev. Sci. Instrum. 82, 113108 (2011); 10.1063/1.3662472

The Effective Fine Structure Constant of Graphene, Measured with Inelastic X-Ray Scattering AIP Conf. Proc. 1267, 106 (2010); 10.1063/1.3482300

Diagnosing direct-drive, shock-heated, and compressed plastic planar foils with noncollective spectrally resolved $\mathrm{x}$-ray scattering

Phys. Plasmas 14, 122703 (2007); 10.1063/1.2819675

Imaging the temporal evolution of nuclear resonant $x$-ray scattering

Appl. Phys. Lett. 78, 2970 (2001); 10.1063/1.1361276

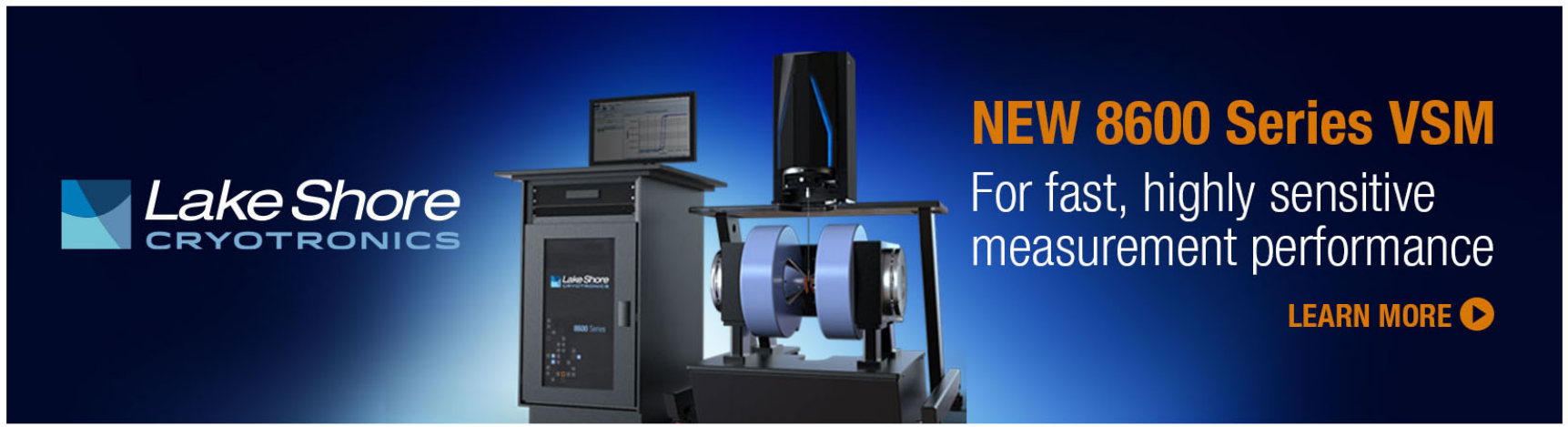




\title{
Tracking the density evolution in counter-propagating shock waves using imaging $X$-ray scattering
}

\author{
U. Zastrau, ${ }^{1,2, a)}$ E. J. Gamboa, ${ }^{2}$ D. Kraus, ${ }^{3}$ J. F. Benage, ${ }^{4,5}$ R. P. Drake, ${ }^{6}$ P. Efthimion, ${ }^{7}$ \\ K. Falk, ${ }^{5,8}$ R. W. Falcone,${ }^{3}$ L. B. Fletcher, ${ }^{2}$ E. Galtier, ${ }^{2}$ M. Gauthier, ${ }^{2}$ E. Granados, ${ }^{2}$ \\ J. B. Hastings, ${ }^{2}$ P. Heimann, ${ }^{2}$ K. Hill, ${ }^{7}$ P. A. Keiter, ${ }^{6}$ J. Lu, ${ }^{7}$ M. J. MacDonald, ${ }^{2,6}$ \\ D. S. Montgomery, ${ }^{5}$ B. Nagler, ${ }^{2}$ N. Pablant, ${ }^{7}$ A. Schropp, ${ }^{9}$ B. Tobias, ${ }^{7}$ D. O. Gericke, ${ }^{10}$ \\ S. H. Glenzer, ${ }^{2}$ and H. J. Lee ${ }^{2}$ \\ ${ }^{1}$ European XFEL GmbH, Holzkoppel 4, 22869 Schenefeld, Germany \\ ${ }^{2}$ SLAC National Accelerator Laboratory, 2575 Sand Hill Road, Menlo Park, California 94025, USA \\ ${ }^{3}$ Department of Physics, University of California Berkeley, Berkeley, California 94720, USA \\ ${ }^{4}$ Sandia National Laboratories, Albuquerque, New Mexico 87185-1189, USA \\ ${ }^{5}$ Los Alamos National Laboratory, Los Alamos, New Mexico 87545, USA \\ ${ }^{6}$ University of Michigan, Ann Arbor, Michigan 48109-2143, USA \\ ${ }^{7}$ Princeton Plasma Physics Laboratory, Princeton, New Jersey 08543-0451, USA \\ ${ }^{8}$ Institute of Physics of the ASCR, ELI-Beamlines, 18221 Prague, Czech Republic \\ ${ }^{9}$ Deutsches Elektronen-Synchrotron DESY, Notkestrasse 85, 22607 Hamburg, Germany \\ ${ }^{10}$ Centre for Fusion, Space and Astrophysics, Department of Physics, University of Warwick, \\ Coventry CV4 7AL, United Kingdom
}

(Received 5 April 2016; accepted 10 July 2016; published online 21 July 2016)

\begin{abstract}
We present results from time-resolved X-ray imaging and inelastic scattering on collective excitations. These data are then employed to infer the mass density evolution within laser-driven shock waves. In our experiments, thin carbon foils are first strongly compressed and then driven into a dense state by counter-propagating shock waves. The different measurements agree that the graphite sample is about twofold compressed when the shock waves collide, and a sharp increase in forward scattering indicates disassembly of the sample 1 ns thereafter. We can benchmark hydrodynamics simulations of colliding shock waves by the X-ray scattering methods employed. Published by AIP Publishing. [http://dx.doi.org/10.1063/1.4959256]
\end{abstract}

The creation of matter with high energy density ${ }^{1}$ in the laboratory is a highly transient process driven by large energy inputs. Consequently, such experiments are often plagued by gradients and fast evolution of the system parameters. On the other hand, many measurements aim at determining equilibrium properties at well-defined conditions to inform modeling of astrophysical objects or technical application of the interaction of intense lasers with matter. One may avoid this dilemma by performing ultra-fast measurements on well-defined states, e.g., isochorically heated matter, ${ }^{2}$ but this approach drastically reduces the phase space that can be probed. Alternatively, one needs to track carefully the evolution of the system, particularly its density and temperature, ${ }^{3}$ to ensure probing of well-defined states or, at least, correctly averaging in time and space.

Compression of solids by shock waves is a frequently used method to produce dense matter at elevated temperatures. ${ }^{4}$ If the shocks are strong enough, warm dense matter or plasma states are created, and the equation of state (EOS) of high pressure materials can be explored. ${ }^{5,6}$ In experiments with strong, single-sided shocks, it is a well-established technique to deduce the system's properties from the measured shock breakout time and particle speed using velocity interferometry (VISAR) at the target rear side. However, many experiments have had to be corrected because of issues related to density inference. ${ }^{7,8}$ Furthermore, VISAR is not applicable for counter-propagating shocks used to reach even

a)ulf.zastrau@xfel.eu higher pressures ${ }^{9}$ and states off the shock Hugoniot. Here, tracking the evolution of spatial and temporal gradients requires the development of innovative diagnostics that ultimately will allow for a much advanced understanding of strongly driven matter.

In this letter, we demonstrate two different X-ray based techniques that can track the density evolution in shockcompressed targets. As a prototypical element, we study carbon which is one of the most abundant elements and plays a crucial role in planetary physics. ${ }^{10}$ The liquid state is only accessible at elevated pressures and temperatures and is especially of interest as it exhibits a very complex electronic structure, ${ }^{11}$ may become conducting, and, thus, contribute to the generation of planetary magnetic fields. ${ }^{12}$ Carbon is also a principle component of the capsule in inertial-confinementfusion experiments ${ }^{13,14}$ where phase transitions ${ }^{15}$ can strongly influence the stability of the capsule.

In the present experiment we use two laser-generated shocks traveling inwards from both sides of a foil. While the shocks propagate separately, hydrodynamics simulations predict typical pressures $\leq 2 \mathrm{Mbar}$ and temperatures $<3 \mathrm{eV}$. When the shocks collide, the predicted pressures rise to $5 \pm 2$ Mbar at temperatures between 5 and $7 \mathrm{eV}$. About $1 \mathrm{~ns}$ later, we experimentally observe a strong disorder in the sample.

The experiments are carried out at the Matter in Extreme Conditions (MECs) ${ }^{16}$ end station at the Linac Coherent Light Source at SLAC National Accelerator Laboratory. Laserdriven ablation creates a dynamic, high pressure state in a graphite foil and, prior to the X-ray experiment, the shock 
compression regime is characterized by established methods. A $5 \mathrm{~ns}$ long flattop pulse from the MEC long-pulse laser at $\lambda=527 \mathrm{~nm}$ wavelength, containing $6 \pm 1 \mathrm{~J}$ energy, is focused onto the front surface of a $115 \mu \mathrm{m}$ rigid graphite $(\mathrm{RG})$ foil with $\rho_{0}=1.8 \mathrm{~g} / \mathrm{cm}^{3}$ mass density. The Gaussian-like irradiation profile (inset in Fig. 1, measured at strong attenuation) has a peak intensity of several $10^{14} \mathrm{~W} / \mathrm{cm}^{2}$ at $20 \mu \mathrm{m}$ full width at half maximum (FWHM) radius, whereas intensities $\sim 10^{12} \mathrm{~W} / \mathrm{cm}^{2}$ are present at radii of $100 \mu \mathrm{m}$.

Under single-sided laser irradiation, the shock can be diagnosed by VISAR looking from the rear side. The laserirradiated side has a $20 \mu \mathrm{m}$ parylene ablator with a $100 \mathrm{~nm}$ aluminum overcoat to prevent penetration of the laser light through the transparent ablator. The rear side is aluminum over-coated and equipped with a $1 \mathrm{~mm}$ LiF VISAR window. We measure a shock transit time of $9.3 \pm 0.2 \mathrm{~ns}$, which yields a maximum 2.2-fold compression and pressures of $P \sim 1.5$ Mbar from the Hugoniot relation. The shock propagation is modeled using two-dimensional hydrodynamics simulations with the code MULTI2D, ${ }^{17}$ in which the EOS is represented by SESAME 7832 table. The simulation agrees with the VISAR results when we assume a larger laser focus with a drive intensity of $\sim 2 \times 10^{13} \mathrm{~W} / \mathrm{cm}^{2}$ in the simulation.

The experimental geometry for counter-propagating shocks employs $115 \mu \mathrm{m}$ thick graphite targets with symmetric $20 \mu \mathrm{m}$ parylene and aluminum coatings on either side, as shown in Fig. 1. Besides rigid graphite (RG), which is a porous polycrystalline system, we study pyrolytic graphite (PG) at a higher initial density of $\rho_{0}=2.2 \mathrm{~g} / \mathrm{cm}^{3}$, which is non-porous and exhibits c-axis order along the surfacenormal.

Counter-propagating shock waves are launched by two nominally identical laser pulses, impinging simultaneously onto opposing sides of the target at $20^{\circ}$ to the target normal (Fig. 1). The target is probed at various time delays at normal incidence by an X-ray laser pulse, where the probed region is centered at the drive laser axis. The X-ray pulses are

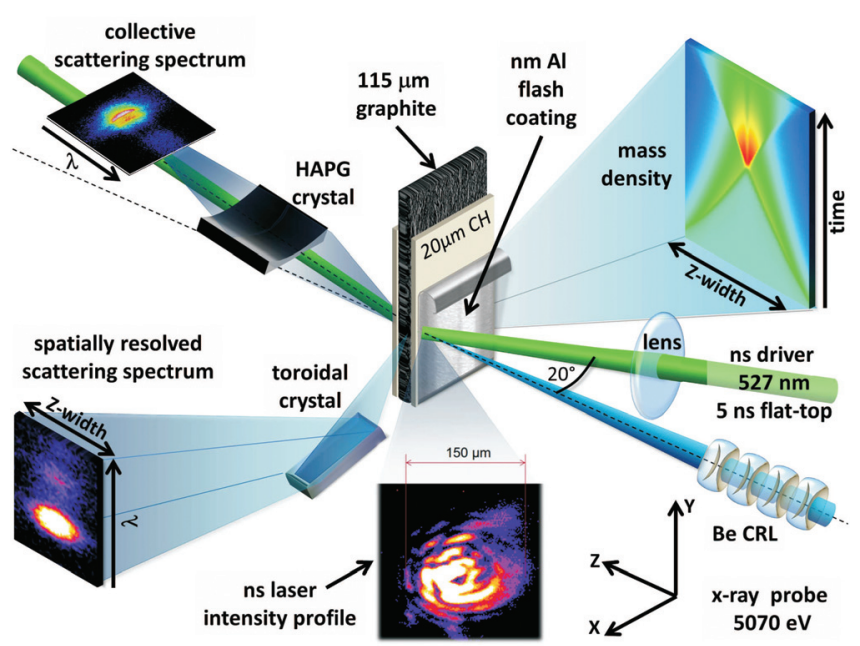

FIG. 1. Experimental setup for counter-propagating shocks. Two laser beams impinge simultaneously onto opposing sides of a graphite foil with angles of $20^{\circ}$ with respect to the target normal. Along the surface normal, and centered to the drive laser foci, an X-ray free-electron laser pulse at $5070 \mathrm{eV}$ photon energy with a diameter of $20 \mu \mathrm{m}$ probes the sample. Two bent crystal spectrometers at scattering angles of $\Theta_{\text {eff }}=29^{\circ}$ and $90^{\circ}$ diagnose the shocked target. linearly polarized, have a photon energy of $5070 \mathrm{eV}, \sim 20 \mathrm{eV}$ bandwidth, and are focused to an area with $20 \mu \mathrm{m}$ diameter. A typical X-ray pulse has a duration of $\sim 50 \mathrm{fs}$. The pulse energy of $2 \mathrm{~mJ}$ has a shot-to-shot variation of up to $\pm 0.5 \mathrm{~mJ}$. The target thickness is about half the $\mathrm{X}$-ray $1 / e$-attenuation length, to minimize absorption.

The counter-propagating shock waves have been simulated using the parameters that reproduced the single-sided VISAR data. Time-dependent profiles of the simulated mass density in PG along the $20 \mu \mathrm{m}$ diameter X-ray beam are shown in the left panel of Fig. 2. For the first 5 ns, the shocks propagate inwards with the density slowly increasing to $3.5 \mathrm{~g} / \mathrm{cm}^{3}$ in PG and $2.9 \mathrm{~g} / \mathrm{cm}^{3}$ in RG, shown by the lines in Fig. 3(b). Typical predicted temperatures (inside the dense target, lines in Fig. 3(c)) stay below $k_{B} T<3 \mathrm{eV}$. When the shocks collide after 5-6 ns, the density is predicted to peak at $4.2 \mathrm{~g} / \mathrm{cm}^{3}$ (PG) and $3.9 \mathrm{~g} / \mathrm{cm}^{3}$ (RG). Simultaneously, the temperature further increases to peak values of $5 \mathrm{eV}(\mathrm{PG})$ and $6 \mathrm{eV}$ (RG).

To diagnose the density evolution, we observed the scattering of the X-ray probe at different time delays up to $9 \mathrm{~ns}$ after the onset of laser irradiation. One-dimensional X-ray imaging perpendicular to shock propagation is achieved by a toroidally curved germanium $\mathrm{Ge}(111)$ crystal spectrometer. ${ }^{18}$ It is positioned $77^{\circ}$ vertically out of the polarization plane to maximize the scattering cross-section $\left(\Theta_{\text {eff }}=90^{\circ}\right.$, wave number transfer $\mathbf{k}=3.63 \AA^{-1}$ ). Coupled to an X-ray charge-coupled device (CCD), the spatial instrument function (top-right panel in Fig. 2) is determined in-situ from imaging the titanium (Ti) $\mathrm{K} \beta$ line $(4932 \mathrm{eV})$ emitted from a few- $\mu \mathrm{m}$ thin Ti foil. It shows a $44 \mu \mathrm{m}$ FWHM on top of a $150 \mu \mathrm{m}$ wide pedestal. A 2.27 -fold magnification is determined by moving this fluorescence source in known steps of $\pm 100 \mu \mathrm{m}$ along the $\mathrm{z}$-axis.

In order to measure diffuse forward scattering at k-values well below the first Bragg peak, a mosaic von-Hámos

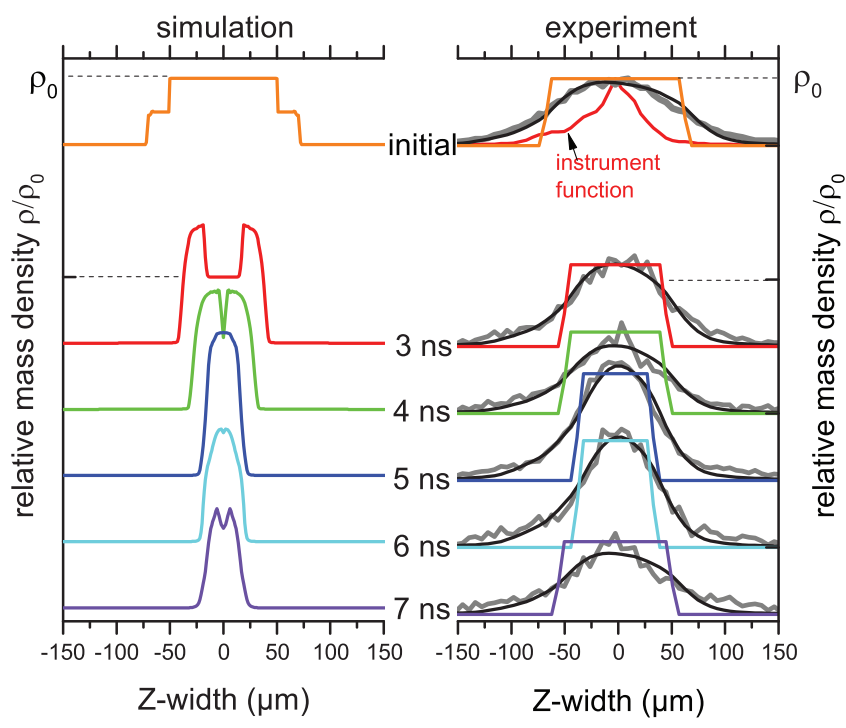

FIG. 2. Left: Hydrodynamics simulations of the mass density evolution along the X-ray beam. Right: Measured scattering Z-profiles of pyrolytic graphite (gray lines), fitted (black lines) by a convolution of the red instrument function (PSF) with rectangular profiles of according widths (colors). All curves are normalized to the initial density $\rho_{0}$ and offset by time delay for presentation. 


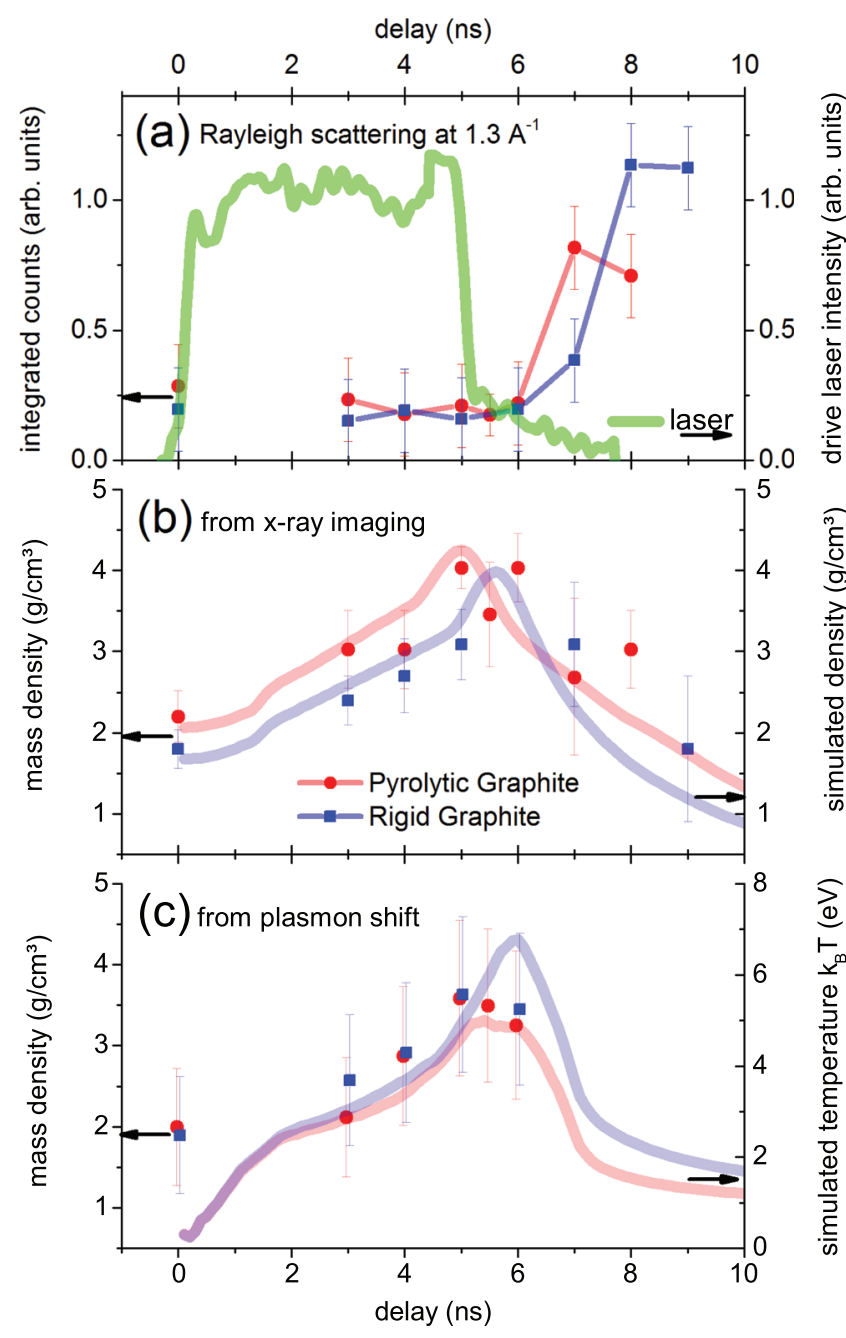

FIG. 3. (a) Measured strength of the forward Rayleigh elastic signal for both pyrolytic and rigid graphite, together with a typical measured temporal laser pulse shape (green curve). (b) The symbols show the density from the $\mathrm{Z}$-width obtained by X-ray imaging. The error bars resemble the deviation between data and fits in Fig. 2, while the lines indicate hydrodynamics simulations of the mass density. (c) The symbols show the mass density from the measured plasmon shift (cf. Fig. 4). Error bars reflect uncertainties in the measured plasmon position, while the lines are hydrodynamic predictions of the temperature evolution.

HAPG crystal spectrometer ${ }^{19}$ is fielded at $24^{\circ}$ vertically while covering $\left(16^{\circ} \pm 6.5^{\circ}\right)$ in the horizontal X-ray polarization plane, yielding an effective scattering angle of $26^{\circ}-32^{\circ}$ (wave number transfer of $\mathbf{k}=1.3 \AA^{-1}$ ). This efficient spectrometer has a spectral resolution of $\Delta E \sim 7 \mathrm{eV}$.

The spectral dispersion is calibrated using Ti $\mathrm{K} \beta$, the vanadium (V) $\mathrm{K} \alpha$ doublet $(4945 \mathrm{eV}, 4952 \mathrm{eV}$ ), and the elastic $\mathrm{X}$-ray scattering $(5070 \mathrm{eV})$. In the observed range, the dispersion of the $\mathrm{Ge}(111)$ imaging spectrometer is approximately linear with $0.372 \mathrm{eV}$ per $13.5-\mu \mathrm{m}$ CCD pixel, while the HAPG spectrometer dispersion corresponds to $0.333 \mathrm{eV}$ per 20- $\mu$ m CCD pixel. Intensity flat-fields are acquired from the continuous thermal emission of a $10 \mu \mathrm{m}$ aluminum foil heated by both ns laser beams.

The right part of Fig. 2 shows peak-normalized Z-profiles (gray) from the imaging spectrometer fitted by a convolution of the spatial instrument function (PSF) with rectangular profiles of appropriate width. Assuming homogeneous densities and a negligible amount of carbon ablation, the lateral compression and hence a relative density change $\rho / \rho_{0}$ is derived. As the initial density $\rho_{0}$ is characterized, this is a measurement of the absolute average density, as the data points in Fig. 3(b) show.

An alternative density measurement uses the densitydependent dynamic response of the valence electrons in forward scattering. The scattering spectra from the HAPG spectrometer show a clear signature of collective plasmon excitation. From Fig. 4, the plasmon energy loss increases from initially $31 \pm 2 \mathrm{eV}$ to a maximum of $\sim 38 \mathrm{eV}$ at $5 \mathrm{~ns}$. Valence electrons can be collectively excited into an available conduction band, oscillating between bonding and antibonding states. In pyrolytic graphite the average direct band gap $\left(\right.$ Penn gap $^{20}$ ) is $\hbar \omega_{\text {Penn }} \sim 12 \mathrm{eV}^{21}$ Thus, the energy loss at a scattering wave vector $\mathbf{k}$ may be modeled as

$$
\hbar \omega(\mathbf{k})=\hbar \sqrt{\omega_{\mathrm{pe}}^{2}+\omega_{\mathrm{Penn}}^{2}}+\frac{\hbar^{2}}{m_{e}} \alpha \mathbf{k}^{2} .
$$

Here, $\hbar=h / 2 \pi$ is the reduced Planck constant and $\omega_{\text {pe }}$ $=\left(e^{2} n_{b} / \varepsilon_{0} m_{e}\right)^{1 / 2}$ is the electron plasma frequency (in this expression, $e$ is the elementary charge, $n_{b}$ is the density of (quasi-)free electrons, $\varepsilon_{0}$ is the dielectric constant, and $m_{e}$ is the electron rest mass). The final quadratic dispersion term goes beyond the usual Drude model and is introduced following the behavior of the random phase approximation for free electrons, ${ }^{22}$ where $\alpha$ is the dispersion coefficient. From the measured energy loss and the Penn gap energy of $12 \mathrm{eV}$, the absolute mass density can be derived via Eq. (1) from the density-dependent electron plasma frequency. In order to reproduce the known initial target density, a dispersion constant of $\alpha=0.35$ is found (both PG and RG have identical density on the nanometer scale). This value is in agreement with recent graphite plasmon data obtained at MEC, showing a trend of $\alpha \sim 0.33$.

Fig. 3(a) shows the strength of diffuse elastic forward scattering that is comparable for both PG and RG. It is constant up to $6 \mathrm{~ns}$ after the onset of laser irradiation (green profile), suggesting that the graphite remains in its initial crystalline state. Subsequently, the amplitude sharply increases by a factor of 4-5 within less than $2 \mathrm{~ns}$. This indicates an abrupt transition from a crystalline to a fluid-like structure.

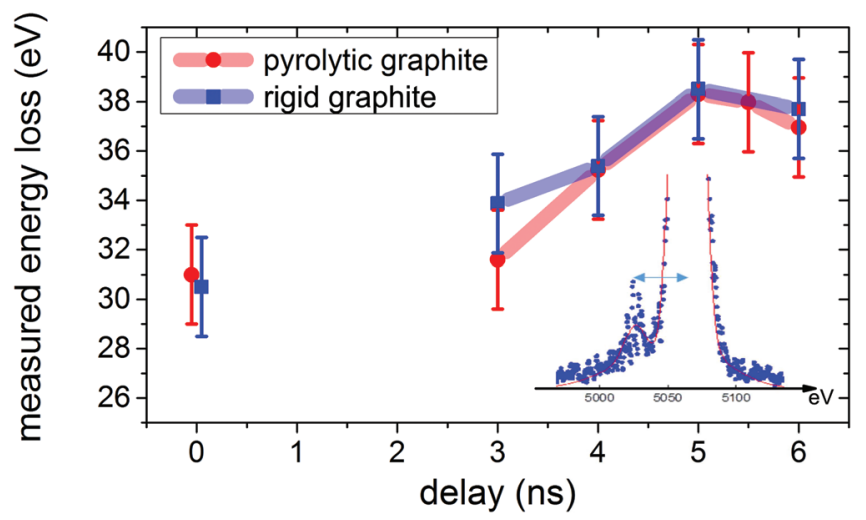

FIG. 4. The measured energy loss (plasmon shift) as a function of delay time. Bottom-right inset: typical elastic scattering spectrum (RG at $6 \mathrm{~ns}$ time delay). Here, the red line is a fit to the raw data (blue dots). 
Figs. 3(b) and 3(c) compare the experimental results from the two density measurements (data points) to the hydrodynamic simulations, shown as semi-transparent lines. These lines are generated by density-weighted integrations along the X-ray path for the mass density and temperature and refer to the right axes. If both the dispersion coefficient and the Penn gap are known, the plasmon-inferred mass density (points in Fig. 3(c)) provides absolute density values. The data points indicate peak compression in both targets at $5 \mathrm{~ns}$, followed by a slight release. However, due to the strong Rayleigh signal (Fig. 3(a)), the plasmon position cannot be determined for delays $>6 \mathrm{~ns}$. Moreover, the Penn gap term in Eq. (1) is valid for the crystalline state, believed to exist for $6 \mathrm{~ns}$, but might change or become metallic $\left(\hbar \omega_{\text {Penn }}=0\right)$ in the fluid state.

Both experimentally obtained densities agree with each other and the simulation within the error bars. Furthermore, the predicted plasma temperature (lines in Fig. 3(c)) correlates with the density evolution. We note that the sharp increase in forward scattering (Fig. 3(a)) is observed $1 \mathrm{~ns}$ after the peak in temperature and density. Here, the target starts to disassemble.

In conclusion, we present two independent time-resolved measurements of the mass density in carbon foils during compression by counter-propagating shock waves. Our measurements, based on X-ray imaging and inelastic collective X-ray scattering, agree and about twofold-compressed graphite is generated when the shock waves collide 5-6ns after the onset of laser irradiation. About $1 \mathrm{~ns}$ later, the drive laser is off and a sharp increase in forward scattering is indicative of a dissembling sample. The measured density evolutions broadly agree with two-dimensional hydrodynamics simulations adjusted with an effective laser drive according to VISAR data from single-sided shock experiments. Our results prove that $\mathrm{X}$-ray scattering yields independent density diagnostics for counter-propagating shock geometries.

Improvements to this method will employ imaging crystals with both improved spatial resolution and magnification. The low scattering cross-section can be overcome by using characteristic fluorescence instead. ${ }^{23}$ Low-Z targets like carbon could be doped by heavier elements-either homogeneously or arranged as tracer layers. In our study, titanium would be a particularly suitable candidate.

We thank D. A. Chapman and J. Vorberger for fruitful discussions and R. Curiel, B. Arnold, and Z. Xing for technical support. This work was performed at the Matter in Extreme Conditions (MEC) instrument of LCLS, supported by the DOE Office of Science, Fusion Energy Science, under Contract No. SF00515. This work was further supported by Fusion Energy Sciences FWP100182. This work was also supported by LCLS, a National User Facility operated by Stanford University on behalf of the U.S. Department of Energy, Office of Basic Energy Sciences. U.Z. and A.S. were supported by the Volkswagen Foundation.

${ }^{1}$ R. P. Drake, High-Energy-Density Physics: Fundamentals, Inertial Fusion, and Experimental Astrophysics (Springer Science \& Business Media, 2006). ${ }^{2}$ P. Patel, A. Mackinnon, M. Key, T. Cowan, M. Foord, M. Allen, D. Price, H. Ruhl, P. Springer, and R. Stephens, Phys. Rev. Lett. 91, 125004 (2003). ${ }^{3}$ P. Kozlowski, B. Crowley, D. O. Gericke, S. P. Regan, and G. Gregori, Sci. Rep. 6, 24283 (2016).

${ }^{4}$ G. Collins, L. Da Silva, P. Celliers, D. Gold, M. Foord, R. Wallace, A. Ng, S. Weber, K. Budil, and R. Cauble, Science 281, 1178 (1998).

${ }^{5}$ R. Smith, J. Eggert, R. Jeanloz, T. Duffy, D. Braun, J. Patterson, R. Rudd, J. Biener, A. Lazicki, A. Hamza et al., Nature 511, 330 (2014).

${ }^{6}$ M. Knudson, M. Desjarlais, A. Becker, R. Lemke, K. Cochrane, M. Savage, D. Bliss, T. Mattsson, and R. Redmer, Science 348, 1455 (2015).

${ }^{7}$ M. Knudson, D. Hanson, J. Bailey, C. Hall, J. Asay, and C. Deeney, Phys. Rev. B 69, 144209 (2004).

${ }^{8}$ D. Hicks, T. Boehly, P. Celliers, J. Eggert, S. Moon, D. Meyerhofer, and G. Collins, Phys. Rev. B 79, 014112 (2009).

${ }^{9}$ L. Fletcher, H. Lee, T. Döppner, E. Galtier, B. Nagler, P. Heimann, C. Fortmann, S. LePape, T. Ma, M. Millot et al., Nat. Photonics 9, 274 (2015).

${ }^{10}$ M. Knudson, M. Desjarlais, and D. Dolan, Science 322, 1822 (2008).

${ }^{11}$ D. Kraus, J. Vorberger, D. O. Gericke, V. Bagnoud, A. Blažević, W. Cayzac, A. Frank, G. Gregori, A. Ortner, A. Otten et al., Phys. Rev. Lett. 111, 255501 (2013).

${ }^{12}$ S. Stanley and J. Bloxham, Nature 428, 151 (2004).

${ }^{13}$ E. Moses, J. Atherton, L. Lagin, D. Larson, C. Keane, B. MacGowan, R. Patterson, M. Spaeth, B. Van Wonterghem, P. Wegner et al., J. Phys.: Conf. Ser. 688, 012073 (2016).

${ }^{14}$ J. Ross, D. Ho, J. Milovich, T. Döppner, J. McNaney, A. MacPhee, A. Hamza, J. Biener, H. Robey, E. Dewald et al., Phys. Rev. E 91, 021101 (2015).

${ }^{15}$ D. Kraus, A. Ravasio, M. Gauthier, D. Gericke, J. Vorberger, S. Frydrych, J. Helfrich, L. Fletcher, G. Schaumann, B. Nagler et al., Nat. Commun. 7, 10970 (2016).

${ }^{16}$ B. Nagler, B. Arnold, G. Bouchard, R. F. Boyce, R. M. Boyce, A. Callen, M. Campell, R. Curiel, E. Galtier, J. Garofoli et al., Synchrotron Radiat. 22, 520-525 (2015)

${ }^{17}$ R. Ramis, J. Meyer-ter Vehn, and J. Ramírez, Comp. Phys. Commun. 180, 977 (2009).

${ }^{18}$ E. Gamboa, P. Keiter, R. Drake, K. Falk, D. Montgomery, and J. Benage, High Energy Density Phys. 11, 75 (2014).

${ }^{19}$ U. Zastrau, A. Woldegeorgis, E. Förster, R. Loetzsch, H. Marschner, and I. Uschmann, J. Instrum. 8, P10006 (2013).

${ }^{20}$ D. Penn, Phys. Rev. 128, 2093 (1962).

${ }^{21}$ L. Calliari, S. Fanchenko, and M. Filippi, Diamond Relat. Mater. 16, 1316 (2007).

${ }^{22}$ S. H. Glenzer and R. Redmer, Rev. Mod. Phys. 81, 1625 (2009).

${ }^{23}$ M. MacDonald, P. Keiter, D. Montgomery, M. Biener, J. Fein, K. Fournier, E. Gamboa, S. Klein, C. Kuranz, H. LeFevre et al., Rev. Sci. Instrum. 85, 11E602 (2014). 